\title{
Oral Tranexamic Acid for the Treatment of Melasma
}

\author{
Karn D, KC S, Amatya A, Razouria EA, Timalsina M
}

Department of Dermatology

Dhulikhel Hospital-Kathmandu University Hospital

Dhulikhel, Kavre, Nepal

Corresponding Author

Dharmendra Karn

Department of Dermatology

Dhulikhel Hospital Kathmandu University Hospital

Dhulikhel, Kavre, Nepal

E-mail: dddkarn@gmail.com

Citation

Karn D, KC S, Amatya A, Razouria EA, Timalsina M. Oral Tranexamic Acid for the Treatment of Melasma. Kathmandu Univ Med J 2012;10(4):40-43.

\begin{abstract}
Background

Melasma poses a great challenge as its treatment is unsatisfactory and recurrence is high. Treatment of melasma using tranexamic acid (oral, topical or intralesional) is a novel concept.
\end{abstract}

\section{Objective}

To compare the efficacy of oral tranexamic acid with routine topical therapies for the treatment of melasma.

\section{Methods}

It is a prospective, interventional, randomized controlled trial conducted among 260 melasma patients. Patients were divided into two groups consisting of 130 patients each. First group (Group A) was given routine treatment measures and oral Tranexamic Acid while second group (Group B) was treated only with routine topical measures. Capsule Tranexamic Acid was prescribed at a dose of $250 \mathrm{mg}$ twice a day for three months and cases were followed for three months. Response was evaluated on the basis of Melasma Assessment Severity Index (MASI). Mean scores between the two groups were then compared.

\section{Results}

Statistically significant decrease in the mean Melasma Assessment Severity Index from baseline to 8 and 12 weeks was observed among group A patients $(11.08 \pm 2.91$ vs $8.95 \pm 2.08$ at week 8 and vs. $7.84 \pm 2.44$ at week $12 ; p<0.05$ for both). While among group $B$ patients the decrease in mean score was significant at 8 weeks and insignificant at 12 weeks follow up $(11.60 \pm 3.40$ vs $9.9 \pm 2.61$ at 8 weeks and vs. $9.26 \pm 3$ at 12 weeks; $p<0.05$ for former but $p>0.05$ for later).

\section{Conclusion}

Addition of oral tranexamic acid provides rapid and sustained improvement in the treatment of melasma.

\section{KEY WORDS}

Melasma, tranexamic acid

\section{INTRODUCTION}

Melasma/cholasma manifests as symmetrical and irregular tan-brown macules on the face. ${ }^{1}$ Though predominantly seen on the malar areas of cheek, it may also appear on forehead and chin. Exposure to ultraviolet (UV) radiation, genetic susceptibility and hormonal imbalance are considered to be its etiological factors. Although no race is spared, melasma is far more common in women and in dark skinned individuals. ${ }^{2}$ It is a common and growing cosmetic concern among Nepalese population with Fitzpatrick skin types III-V. ${ }^{3}$

Till date none of its existing treatment modalities has provided quick and sustained result. Today topical hydroquinone is considered to be the gold standard among topical treatments of melasma. ${ }^{4}$ Prevention of UV radiation, topical bleaching agents, chemical peel and 
light based therapies are the currently existing plethora of remedies. Addition of Tranaxemic Acid (TXA) for the treatment of melasma is a novel concept. Previously used as an antifibrinolytic agent, TXA is recently found to inhibit plasminogen-keratinocyte interaction decreasing the tyrosinase activity causing decreased melanin synthesis from the melanocytes. ${ }^{5,6}$ Very few clinical trials have been conducted regarding the efficacy of TXA (oral, topical or intralesional) for the treatment of melasma. This study is thus conducted to compare the efficacy of oral TXA along with routine treatment measures for the treatment of melasma.

\section{METHODS}

It is a prospective, interventional, randomized controlled trial conducted among 260 (217 female and 43 male) melasma patients. The sample size was calculated at 236 taking prevalence of $10 \%$ in Nepalese population with confidence level at $95 \%$ and margin of error at $0.05 .{ }^{16}$ This study was conducted in the Department of Dermatology, Dhulikhel Hospital, Dhulikhel, Nepal from June 2011 to June 2012, after taking consent from the institutional review committee. Age of patients ranged from $17-55$ years (mean age $30.3 \pm 9.01$ years). All patients were checked for bleeding time, clotting time, platelet count before they were included in the study. Patients with abnormal parameters were not included in the study. The type of melasma was graded with Wood's lamp and dermatoscopic examination. Patients were randomly divided into two groups consisting of 130 patients each using random number table. First group (Group A) was added on with oral TXA along with routine topical treatment measures while second group (Group B) was treated only with routine topical measures. Topical treatment included topical hydroquinone and sunscreen according to the skin types. Capsule tranexamic acid (Trancap ${ }^{\circledR}$ ) was prescribed at a dose of $250 \mathrm{mg}$ twice a day for three months and cases were followed up four weekly till the end of third month. Patients who discontinued the treatment or wanted chemical peel or laser for better response were excluded from the study. Response was evaluated on the basis of Melasma Assessment Severity Index (MASI) score. ${ }^{7} \mathrm{MASI}$ score was calculated during the start of therapy (baseline) and at the end of eight and twelve weeks.

MASI is a scoring system introduced by Kimbrough-Green CK et. al. used to quantify the severity of melasma. ${ }^{7}$ Four areas of the face are evaluated: forehead (F), right malar region $(\mathrm{MR})$, left malar region $(\mathrm{ML})$ and chin $(\mathrm{C})$, corresponding to $30 \%, 30 \%, 30 \%$, and $10 \%$ of the total face respectively. Amount of pigmentation involved by melasma in these four areas ( $A F, A M R, A M L$, and $A C)$ is graded as a numerical value: 0 : no involvement; 1 : less than $10 \%$ involvement; 2 : 10-29\%; 3: 30-49\%; 4: 50-69\%; 5: 70-89\%; and 6: $90-100 \%$. Severity of melasma is graded upon two factors; darkness (D) of melasma compared to the normal skin and homogeneity $(\mathrm{H})$ of hyperpigmentation. These are assessed on a scale from 0 to 4 . The rating scale for both darkness and homogeneity of melasma is as follows; 0 : absent; 1 : slight; 2 : mild; 3: marked; and 4: maximum. MASI score is then calculated according to the following formula:

$\mathrm{MASI}=0.3(\mathrm{DF}+\mathrm{HF}) \mathrm{AF}+0.3(\mathrm{DMR}+\mathrm{HMR}) \mathrm{AMR}+0.3(\mathrm{DML}$ $+\mathrm{HML}) A M L+0.1(D C+H C) A C$.

The maximum score for MASI can be 48 while minimum can be 0 . SPSS ver 16 was used for the statistical analysis. Mean MASI scores were compared with students' T-test and statistical significance was compared. Patient satisfaction score was subjectively graded on the basis of four point Likert scale: excellent, good, fair, and poor. Possible side effects of TXA were noted on follow up of patients.

\section{RESULTS}

Table 1. Gender of patients, pathological type and distribution of melasma.

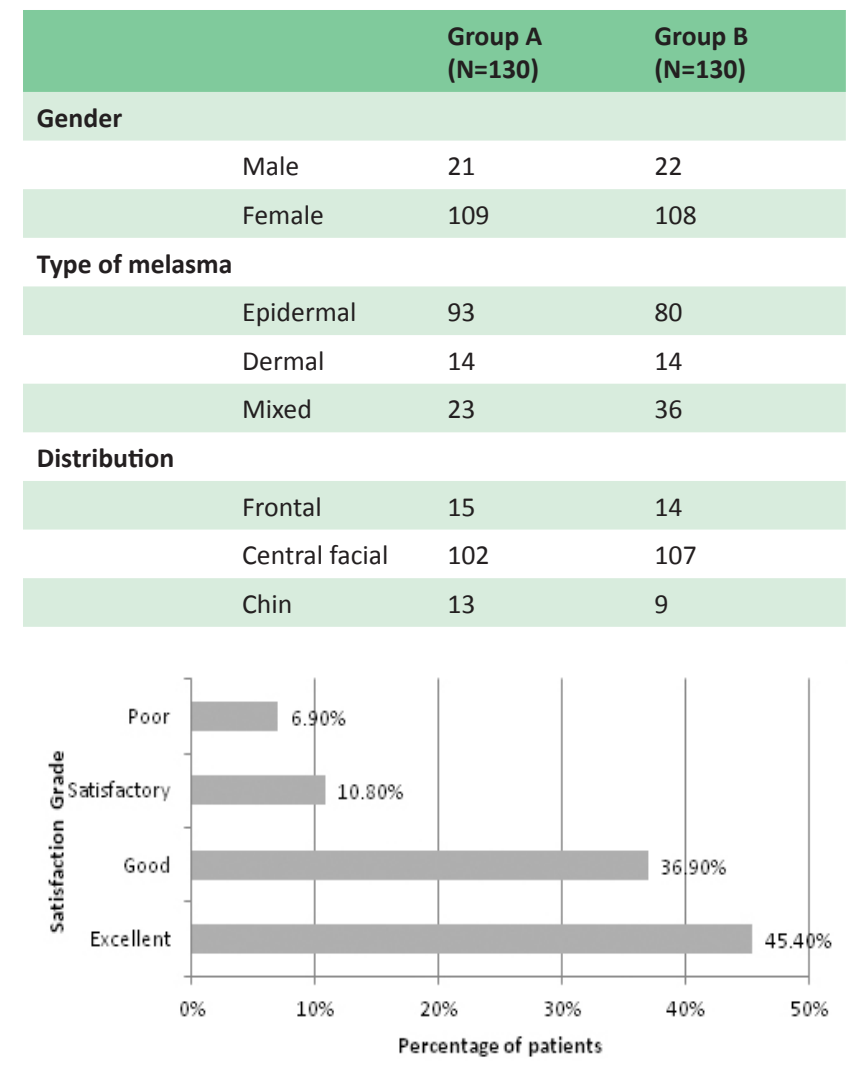

Fig 1. Patient satisfaction score for group A patients.

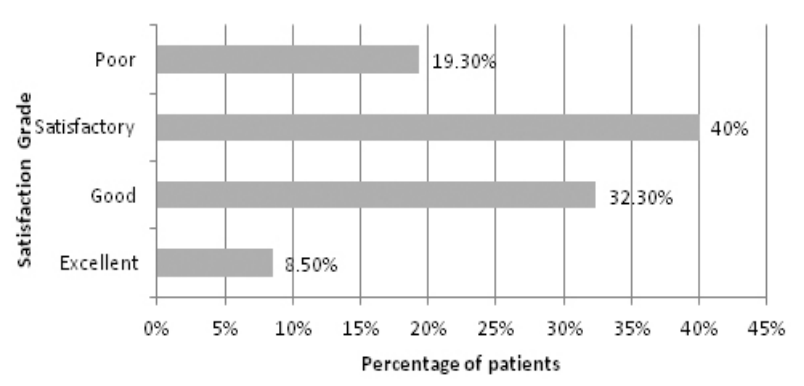

Fig 2. Patient satisfaction score for group B patients. 


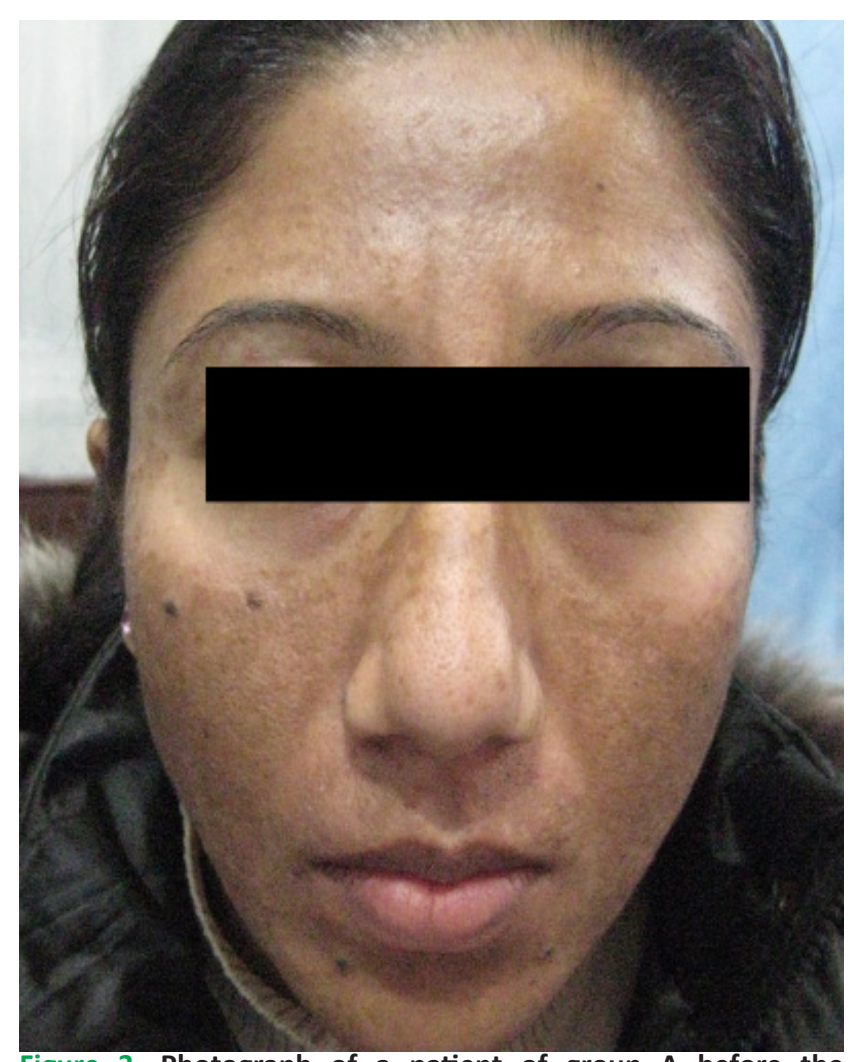

Figure 3. Photograph of a patient of group $A$ before the treatment.

The demographical variables, type and distribution of melasma is shown in table 1 . Patients rating for good to excellent outcome accounted $82.3 \%$ and $40.8 \%$ among group A and group B patients respectively. (Fig. 1,2).

Among group A patients, statistically significant decrease in the mean MASI score was observed from baseline at eight and twelve weeks $(11.08 \pm 2.91$ vs $8.95 \pm 2.08$ at week 8 and vs. $7.84 \pm 2.44$ at week $12 ; p<0.05$ for both). While among group $B$ patients the decrease in mean score was significant at eight weeks and insignificant at twelve weeks follow up $(11.60 \pm 3.40$ vs $9.9 \pm 2.61$ at eight weeks and vs. $9.26 \pm 3$ at 12 weeks; $p<0.05$ for former but $p>0.05$ for later). Fig 3 and 4 shows photograph of a patient of group a before and after the treatment.

Oral TXA was found to be relatively safe. Certain side effects seen among group A patients were oligomenorrhea (14.7\% ladies), belching (9.2\%), abdominal cramps (6.9\%), palpitation and urticarial rash with angioedema (in one patient each). No serious systemic complication was observed during the study period.

Two patients treated for hydroquinone induced exogenous ochronosis with TXA, topical retinoids and non hydroquinone bleaching agent were found to have significant improvement at the end of 12 weeks follow. Our experience also showed that response of epidermal melasma was quicker and better than compared to dermal or mixed variant. Further, some patients of group A excluded from the study during middle of the treatment were simultaneously treated with ablative fractional Erb:

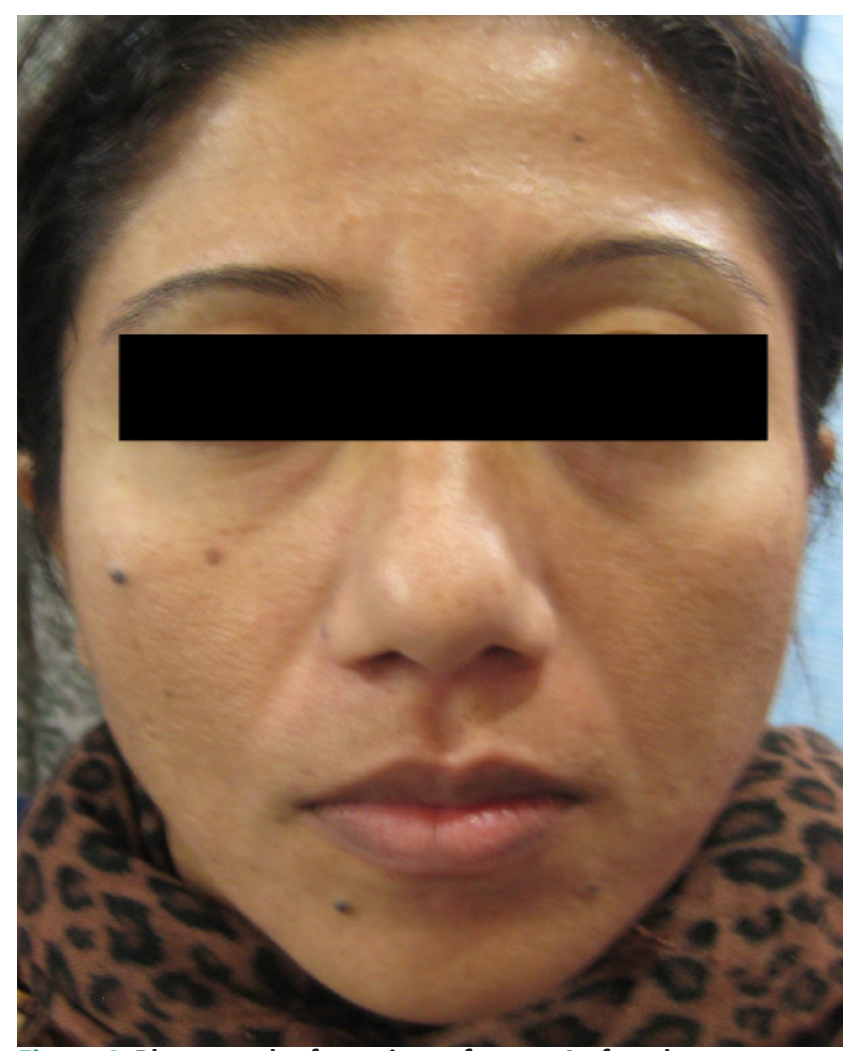

Figure 4. Photograph of a patient of group A after the treatment.

YAG laser and were found to have better and quicker response in dermal or mixed type of melasma.

\section{DISCUSSION}

Pathologically increased epidermal melanogenesis following a triggering factor results in melasma. ${ }^{1}$ Measurement of endocrine parameters have shown elevated levels of luteinizing hormone and decreased levels of oestradiol, suggesting subclinical mild ovarian dysfunction. ${ }^{2}$ The major target of its treatment thus remains to decrease the melanin synthesis, remove the excess melanin or use of bleaching agents and avoidance of any etiological agent. Treatment of melasma is a challenge because of its multifactorial etiology. Topical treatments may temporarily result in improvement, but the condition often returns.

Clinical trials have shown that topical medications demonstrate some efficacy in the epidermal type but not in dermal or mixed type of melasma. 8 .9 Prolonged application, slow response, limited effects and undesirable recurrence are the major disadvantages of topical therapies causing patients to abandon the treatment. Also, topical bleaching agents may irritate the skin and develop postinflammatory hyperpigmentation or result in exogenous ochronosis. Laser assisted treatment is a good method however recurrence remains disappointing. ${ }^{10}$ The most effective and safe treatment for melasma is thus yet to be explored.

Tranexamic acid (trans-4-aminomethyl cyclohexane 
carboxylic acid) is used as a haemostatic agent due to its antifibrolytic action. ${ }^{11}$ It is a synthetic derivative of the amino acid lysine used to treat and prevent excessive bleeding. Nijor first studied and reported the action of TXA on melasma in 1979. ${ }^{12}$ However, limited studies are found in the literature regarding the use of TXA on melasma. Maeda $\mathrm{K}$ et. al. studied the role of TXA in human melanocyte and keratinocyte cultures. ${ }^{11,12}$ Their results revealed that TXA inhibits melanin synthesis in epidermal melanocytes' tyrosinase activity by blocking the interaction of melanocytes and keratinocytes by inhibition of plasminogen/plasmin system. TXA acts by attaching to the lysine-binding sites of plasmin and plasminogen and also prevents ultraviolet rays induced pigmentation.

Similar study as ours was conducted on a split-face based trial with topical TXA gel. ${ }^{13}$ Their results showed lightening of pigmentation but was insignificant in comparison to the control group. Also topical TXA produced erythema. Lee JH et al. injected localized intradermal microinjection of TXA for Melasma and found statistically significant lightening. ${ }^{9}$ Histological evaluation following oral and topical TXA for melasma was done which concluded that TXA decreases epidermal pigmentation associated with melasma and also reverses melasma-related dermal changes, such as vessel number and increased numbers of mast cells. ${ }^{14}$ Use of oral TXA for melasma in similar dose have been tried in Chinese population and authors recommend TXA to be an effective and safe therapy for the treatment of melasma. ${ }^{15}$ Dose of oral TXA used in melasma is far less than that prescribed for its haemostatic action. Venous thromboembolism,

\section{REFERENCES}

1. Anstey AV. Disorders of Skin Color. In: Burns T, Breathnach S, Cox N, Griffiths C eds. Rook's Textbook of Dermatology. 8th ed. WileyBlackwell; 2010. p. 58.34

2. Katsambas AD, Stratigos AJ, Lotti TM. Melasma. In: Andreas DK, Lotti TM eds. European Handbook of Dermatological Treatments. 2nd ed. Springer; 2003. p. 336.

3. Karn D, Khatri R, Timalsina M. Prevalence of skin diseases in Kavre district, Nepal. Nepal I Dermatol Venereol Leperol 2010; 10:07-10.

4. Grimes PE. Management of hyperpigmentation in darker racial ethnic groups. Semin Cutan Med Surg 2009;28:77-85.

5. Maeda K, Tomitab Y. Mechanism of the inhibitory effect of tranexamic acid on melanogenesis in cultured human melanocytes in the presence of keratinocyte-conditio-ned medium. J Health Sci 2007; 53:389-96.

6. Maeda K, Naganuma M. Topical trans-4aminomethylcyclohexanecarboxylic acid prevents ultraviolet radiation-induced pigmentation. I Photochem Photobiol B.1998;47:136-41.

7. Kimbrough-Green CK, Griffiths CEM, Finkel LJ, Hamilton TA, BulengoRansby SM. Topical retinoic acid (tretinoin) for melasma in black patients. Arch Dermatol 1994;130:727-733.

8. Ejaz A, Raza N, Iftikhar N, Muzzafar F. Comparison of $30 \%$ salicylic acid with Jessner's solution for superficial chemical peeling in epidermal melasma. J Coll Physicians Surg Pak 2008;18:205-08. myocardial infarction, cerebrovascular accidents and pulmonary embolism are some reported complications in haemostatic dose of TXA. It is contraindicated for patients having acquired defective color vision, an active intravascular clotting condition, and hypersensitivity to TXA. Hence, proper patient selection ruling out any risk factor resulting in hypercoagulability is of pivotal importance prior to the start of therapy.

Our results correspond with the related studies where successful lightening was observed following oral TXA administration. Despite the lack of an objective measurement in the improvement, we observed good clinical outcome of melasma. Further, longer duration follow up and randomized controlled trials involving larger number of patients are demanded for further exploration of efficacy of TXA in the near future. Oral TXA being a non invasive, nonirritating drug with low side effect profile we routinely recommend the use of low dose oral TXA in patients with melasma.

The study is done in a single center over a period of one year. A much larger multicenter study is necessary to validate the study.

\section{CONCLUSION}

Addition of oral TXA to routine treatment measures provides a rapid and better lightening in patients with melasma. Low dose oral TXA is thus recommended for the treatment of melasma.

9. Lee JH, Park JG, Lim SH, KIM JY. Localized Intradermal Microinjection of Tranexamic Acid for Treatment of Melasma in Asian Patients: A Preliminary Clinical Trial. Dermatol Surg 2006;32:626-31.

10. Karn D, KC S, Amatya A, Razouria EA, Timalsina M et. al. Q-Switched Neodymium-Doped Yttrium Aluminum Garnet Laser Therapy for Pigmented Skin Lesions: Efficacy and Safety. Kathmandu Univ Med Jr 2012;38:46-50.

11. Dunn CJ, Goa KL. Tranexamic acid: a review of its use in surgery and other indications. Drugs 1999;57:1005-32.

12. Nijor T. Treatment of melasma with tranexamic acid. Clin Res 1979;13:3129-31.

13. Ayuthaya PKN, Niumphradit N, Manosroi A, Nakakes A. Topical $5 \%$ tranexamic acid for the treatment of melasma in Asians: A doubleblind randomized controlled clinical trial. J Cosmet Laser Ther 2012;14:150-54.

14. Na JI, Choi SY, Yang SH, Choi HR, Kang HY, Park KC. Effect of tranexamic acid on melasma: a clinical trial with histological evaluation. J Eur Acad Dermatol Venereol 2012.

15. Wu S, Shi H, Wu H, Yan S, Guo J, Sun Y. et. al. Treatment of Melasma With Oral Administration of Tranexamic Acid. Aesthetic Plast Surg 2012;36:964-70.

16. Walker SL, Shah M, Hubbard VG, Pradhan HM, Ghimire M, Skin Disease is Common in Rural Nepal : results of a point prevalence study . British Journal of Dermatology 2008; 158: 334-38. 